Full Paper: The effect of polymer polydispersity on the polymer-induced interaction between colloidal particles due to non-adsorbing ideal chains is investigated. An analytical theory is developed for the polymer-segment density between two plates and in the space surrounding two spheres by extending a recently proposed superposition approximation to include polymer polydispersity. Monte Carlo computer simulations were made to test the validity of the analytical theory. The polymer densities predicted by the superposition approximation are in reasonable agreement with simulation results for the polydisperse case. The simulations show that depletion leads to a size fractionation of the polymers. It is shown that size polydispersity has a small effect on the interaction between two parallel plates but a more significant effect on the interaction between two spheres. The range of the potential increases and the contact potential drops with increasing polydispersity.

Polymer-segment density as a function of $y$ for three values of $x$, as indicated, in the space surrounding two colloidal spheres with radius $R=R_{\mathrm{g} 0}$ and $h=0.48 R_{\mathrm{g} 0}$. Symbols are the MC results: polydisperse polymer $(O ; z=1)$ and monodisperse polymer (O) samples. Curves are the predictions of the product-function approximation for monodisperse polymer (solid lines) and polydisperse polymer $(z=1$, dashed lines).

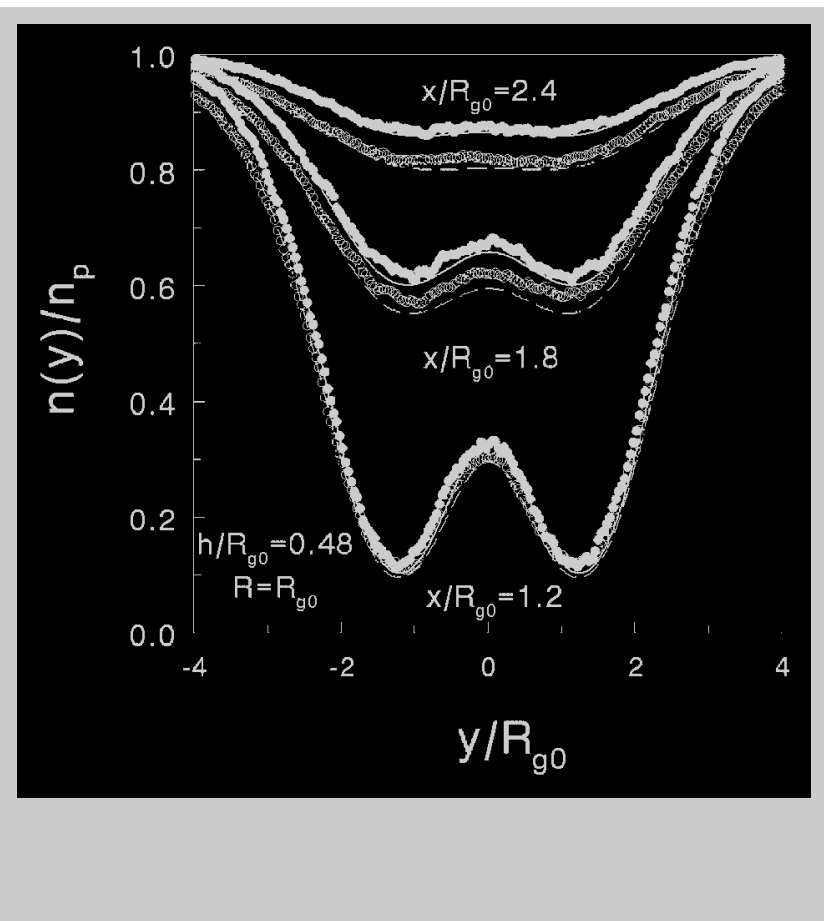

\title{
Polymer Polydispersity Effect on Depletion Interaction between Colloidal Particles
}

\author{
Remco Tuinier, ${ }^{* 1}$ Andrei V. Petukhov ${ }^{2}$ \\ ${ }^{1}$ Forschungszentrum Jülich, Institut für Festkörperforschung, Weiche Materie, D-52425 Jülich, Germany \\ E-mail: r.tuinier@fz-juelich.de \\ ${ }^{2}$ Van't Hoff Laboratory, Debye Research Institute, University of Utrecht Padualaan 8, $3584 \mathrm{CH}$ Utrecht, The Netherlands
}

Keywords: colloids; density; Monte Carlo simulation; polydispersity; polymer interactions; size fractionation

\section{Introduction}

The polymer-mediated depletion interaction between colloidal particles has been the subject of many theoretical and experimental studies, especially over the last decade (for a recent review $\left.s^{[1]}\right)$. In most studies the polymers are treated as monodisperse, although in practice they are polydisperse in size, even in experimental model studies. ${ }^{[2-5]}$ Incorporation of the size polydispersity of polymers has gained very limited attention in (polymer-induced) depletion theories. So far, polydisperse polymers were simplified as polydisperse spheres. ${ }^{[6-9]}$ However, an extension towards more realistic polymer models involving polydispersity is required in order to take packing effects of long polymers around small spheres into account and properly predict polydispersity effects on the interaction potential.

Two powerful tools to calculate the interaction between colloidal particles, attributable to non-adsorbing polymers, smaller colloidal particles, or rods, are the force method (see for instance Mao et al. ${ }^{[10]}$ ) and the adsorption method. ${ }^{[11]}$ The force method focuses on the interaction between two plates immersed in a solvent containing the other component by calculating the osmotic pressure between the plates and outside the plates. Integration then yields the interaction potential between the plates. More relevant for practical systems is the interaction between two spheres immersed in a solution containing polymer chains. Here, the force method can be used only in the Derjaguin limit of infinitely large spheres. The adsorption method is 
another useful thermodynamic tool and is briefly explained in the next section. Previously, the adsorption method was used to calculate the polymer-mediated interaction between two spheres ${ }^{[12]}$ and between a sphere and a plate. ${ }^{[13]}$ In order to be able to use the adsorption method, the (negative) adsorption of polymer segments in the space surrounding the two colloidal particles is required. The product-function approximation $^{[12]}$ was shown to predict the complex polymer-segment density of ideal polymer chains between the particles very accurately. It assumes that the total polymer-segment density, normalized with the bulk polymer-segment density, equals the product of the polymer densities generated by the individual colloidal particles. Here we extend the adsorption method with the productfunction approximation to calculate the interaction between particles induced by polydisperse ideal polymer chains.

As a starting point, an exact result for the interaction between two plates immersed in an ideal solution of polydisperse polymer chains will be derived and compared with Monte Carlo computer simulation results. Next, the results from the computer simulations and the analytical theory are compared on the level of the local polymersegment concentrations between two parallel plates and surrounding two spheres. Finally the interaction between two spheres is calculated and compared with Monte Carlo simulation results.

\section{Polydisperse Ideal Polymer between Two Parallel Plates}

\section{Force Method}

Asakura and Oosawa ${ }^{[14]}$ derived an exact expression for monodisperse ideal polymer chains confined between two parallel plates using the force method. The force acting on the plates is the difference of the pressure between the plates, $\Pi_{\mathrm{i}}$, and outside the plates, $\beta \Pi_{\mathrm{o}}=n_{\mathrm{b}}$, where $n_{\mathrm{b}}$ is the bulk polymer concentration and $\beta=1 / \mathrm{k} T$. The pressure between the plates can be calculated from the partition function $Z$

$$
\beta \Pi_{\mathrm{i}}=\left(\frac{\partial \ln Z}{\partial V}\right)_{T}
$$

where $Z$ equals

$$
Z=\frac{(V \chi)^{n_{\mathrm{b}} V}}{\left(n_{\mathrm{b}} V\right) !}
$$

with $h$ the plate-plate distance. The term $\chi$ reads

$$
\chi=\frac{8}{\pi^{2}} \sum_{p=1,3,5, \ldots} \frac{1}{p^{2}} \exp \left(-\frac{p^{2} \pi^{2} N}{6 h^{2}}\right)
$$

where $N$ is the number of links between the segments and is related to the radius of gyration of ideal chains as $R_{\mathrm{g}}=\sqrt{ }(N / 6)$. The partition function was calculated by Asakura and Oosawa ${ }^{[14]}$ by solving the diffusion equation for ideal polymers

$$
\frac{\partial G_{N}\left(\underline{r} ; \underline{r}^{\prime}\right)}{\partial N}=\frac{1}{6} \Delta_{\underline{r}} G_{N}\left(\underline{r} ; \underline{r}^{\prime}\right)
$$

with the boundary condition that the polymer-segment concentration vanishes at the plates. The function $G_{N}\left(\underline{r}, \underline{r}^{\prime}\right)$ describes the probability of finding the $N^{\text {th }}$ segment of the polymer chain at position $\underline{r}^{\prime}$ while its origin lies at $\underline{r}$. The force can now easily be calculated ${ }^{[12]}$ and integration of the force yields the interaction potential $W(h)$

$$
\frac{\beta W_{\mathrm{pl}}(h)}{n_{b}}=-\chi h+h-2 \Delta
$$

where the proper boundary condition $W(\infty)=0$ was used, recovering the depletion layer thickness per plate, $\Delta$, being $2 R_{\mathrm{g}} / \sqrt{ } \pi\left([h-\chi \mathrm{h}] \rightarrow 4 R_{\mathrm{g}} / \sqrt{ } \pi\right.$ for $\left.h \rightarrow \infty\right)$. The result of Equation (5) is plotted in Figure 1 as the solid curve ('monodisperse').

For two plates immersed in a polydisperse polymer solution the same analysis can be performed, also leading to Equation (5), but with different functions $\chi$ and $\Delta$. The function $\chi_{\text {pol }}$ reads

$$
\chi_{\text {pol }}=\frac{\int_{0}^{\infty} \chi(N) \Psi(N) N \mathrm{~d} N}{\int_{0}^{\infty} \Psi(N) N \mathrm{~d} N}
$$

which replaces $\chi$ in Equation (5) and where $\Psi(N)$ is the chain length distribution. The polydisperse depletion

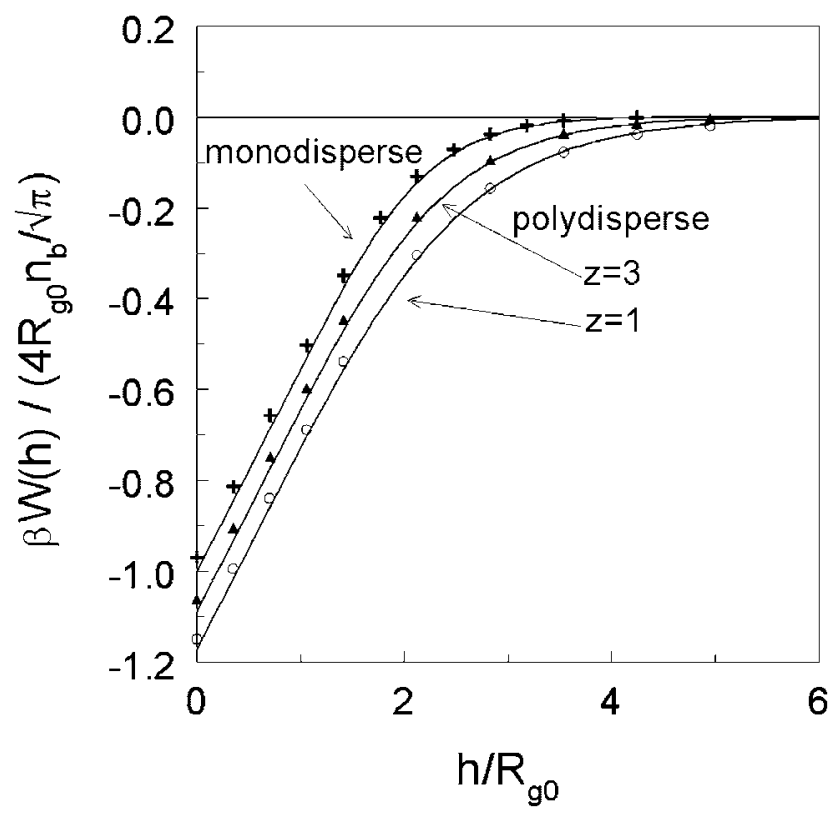

Figure 1. Interaction potential between two parallel plates $W(h)$ as a function of the distance between the plates, $h$. Solid curves are exact results (Equation (5)) for monodisperse and polydisperse ideal polymers with Schulz $z$ parameters as indicated. Symbols refer to the MC simulation results. $\beta=1 / \mathrm{k} T$. 
thickness, $\Delta_{\text {pol }}$, becomes (see also appendix):

$$
\Delta_{\text {pol }}=\frac{\int_{0}^{\infty} \sqrt{\frac{2 N}{3 \pi} \Psi(N) N \mathrm{~d} N}}{\int_{0}^{\infty} \Psi(N) N \mathrm{~d} N}
$$

As the chain length distribution we choose the Schulz distribution

$$
\Psi(N)=\frac{1}{\Gamma(z+1)}\left(\frac{z+1}{N_{0}}\right)^{z+1} N^{z} \exp \left(-\left(\frac{z+1}{N_{0}}\right) N\right)
$$

where $N_{0}$ is the number-averaged chain length and $z$ reflects the width of the distribution; the fractional polydispersity equals: $\frac{1}{\sqrt{z+1}}$.

A $z$ parameter of 1 refers to a quite polydisperse distribution having a standard deviation close to $70 \%(z=3 \mathrm{ca}$. $50 \%$ ). The result for the interaction between two plates due to polydisperse ideal polymers with a Schulz distribution are also plotted in Figure 1 for $z=1$ and $z=3$ (solid curves; 'polydisperse'; Schulz, $z=1, z=3$ ). In comparison with the result for the monodisperse distribution we find that polydispersity makes the attraction stronger. It follows that:

$$
\frac{W_{\mathrm{pl}}^{\mathrm{poly}}(0)}{W_{\mathrm{pl}}(0)}=\frac{\Delta \sqrt{\pi}}{2 R_{\mathrm{g}}} \frac{\Delta_{\text {poly }}}{\Delta_{\text {mono }}}
$$

The effect of polydispersity is quite small; for a polydisperse polymer solution having a standard deviation in chain length of $70 \%(z=1$; to compare with experimental results: $\left.\bar{M}_{\mathrm{w}} / \bar{M}_{\mathrm{n}} \approx 1.5\right)$ the attraction increases with a factor of only 1.17. Further it can be noted that the range of the attraction also increases with increasing polydispersity.

\section{Adsorption Method}

The main interest is on the depletion interaction between two spheres in a solution with polydisperse ideal chains. Therefore we use the adsorption method, which is first applied to the case of two parallel flat plates, in order to compare with exact results for polydisperse ideal polymers derived in section Force Method. The adsorption method $^{[11-13]}$ relates the adsorption $\Gamma(h)$ to the interaction potential $W(h)$ which for ideal polymers leads to:

$$
\beta W(h)=\Gamma(\infty)-\Gamma(h)
$$

The adsorption itself is the volume integral over the polymer-segment concentration profile $n(\underline{r})$

$$
\Gamma(h)=n_{\mathrm{b}} \int_{V} \mathrm{~d} r\left\{\frac{n(\underline{r})}{n_{\mathrm{p}}}-1\right\}
$$

where $n_{\mathrm{p}}$ is the polymer-segment concentration in the bulk $\left(=N n_{\mathrm{b}}\right)$. Hence the focus is now on the calculation of the local polymer-segment concentration $n(\underline{r})$ (further denoted as polymer-segment density). Previously, it was shown that the product function Ansatz ${ }^{[12]}$

$$
\frac{n(\underline{r})}{n_{\mathrm{p}}}=f_{1}(\underline{r}) f_{2}(\underline{r})
$$

where $f_{j}(\underline{r})$ is the profile generated by particle $j$ at position $\underline{r}$, gives a very good description of the polymer-segment density between two parallel plates, ${ }^{[12]}$ two spheres,,${ }^{[12]}$ and between a sphere and a plate, ${ }^{[13]}$ as compared with computer simulations and accurately describes the interaction potential. For the interaction between two parallel plates we thus need the normalized polymer-segment density, $f_{\mathrm{p}}(x)=n(x) / n_{\mathrm{p}}$, near a single plate which has been calculated by Eisenriegler ${ }^{[15]}$

$$
\begin{aligned}
f_{\mathrm{p}}(x) & =2 \operatorname{erf}\left(\frac{x}{2 R_{\mathrm{g}}}\right)-\operatorname{erf}\left(\frac{x}{R_{\mathrm{g}}}\right) \\
& +\frac{2}{\sqrt{\pi}}\left(\frac{x}{R_{\mathrm{g}}}\right)\left[\exp \left(-\left(\frac{x}{2 R_{\mathrm{g}}}\right)^{2}\right)-\exp \left(-\left(\frac{x}{R_{\mathrm{g}}}\right)^{2}\right)\right] \\
& +2\left(\frac{x}{R_{\mathrm{g}}}\right)^{2}\left[\frac{1}{2}-\operatorname{erf}\left(\frac{x}{R_{\mathrm{g}}}\right)+\frac{1}{2} \operatorname{erf}\left(\frac{x}{2 R_{\mathrm{g}}}\right)\right]
\end{aligned}
$$

which thus only depends on the distance from the surface $x$ and the chain length $N\left(=6 R_{\mathrm{g}}^{2}\right)$. For polydisperse polymers, with a chain length distribution $\Psi(N)$, the polymer segment density near a single flat plate reads:

$$
f_{\mathrm{p}}^{\text {poly }}(x)=\frac{\int_{0}^{\infty} \mathrm{d} N \Psi(N) N f_{\mathrm{p}}(x, N)}{\int_{0}^{\infty} \mathrm{d} N \Psi(N) N_{\mathrm{p}}}
$$

In Figure 2 the resulting polymer densities according to Equation (14) (with (13) for $f_{\mathrm{p}}$ ) are plotted for a Schulz distribution with $z=3$ and $z=1$ as the full curves. The monodisperse result (Equation (13)) is plotted as well (from top to bottom: monodisperse, $z=3, z=1$ ). The distance from the surface is normalized with $R_{\mathrm{g} 0}$, which is defined as $\sqrt{ }\left(N_{0} / 6\right)$. Distances and positions are normalized with $R_{\mathrm{g} 0}$ throughout this paper. In order to later test the productfunction approximation (Equation (12)) the results will be compared with Monte Carlo computer simulations.

The simulation of the density profile of polymer segments between two flat walls was made by mapping the problem onto a one-dimensional lattice model with a uniform grid of lattice points. One Monte Carlo run consisted of performing typically $10^{6}$ one-dimensional random walks. The starting lattice point was set at random between the two parallel flat plates and the chain length was fixed for monodisperse polymers or was randomly generated using the Schultz distribution for the polydisperse case. Typically, we took a (number-averaged) chain length, $\langle N\rangle$, of 400 segments. Since the walks in the two orthogonal directions (parallel to the plates) are not performed, the radius of gyration in one dimension, $R_{\mathrm{g}}^{1 \mathrm{D}}$, equals $\sqrt{ }\left\{\left\langle N_{1 \mathrm{D}}\right\rangle / 2\right\}$. We have verified that the chain length 


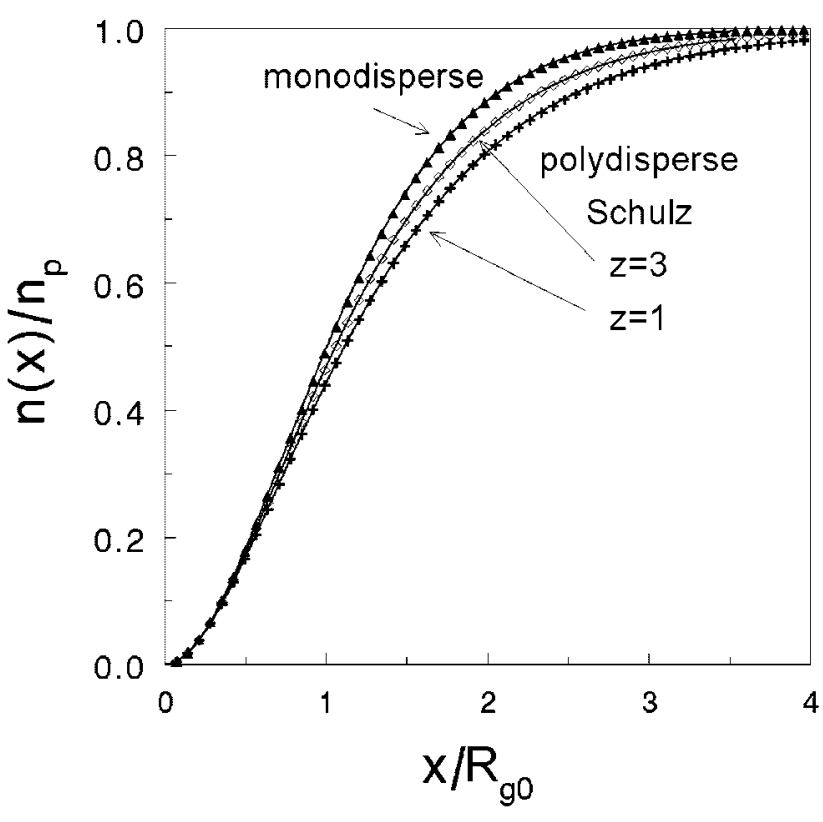

Figure 2. Polymer-segment density of mono- and polydisperse ideal polymers in solution near a single flat plate. Monte Carlo computer simulation results are given by the symbols $(\boldsymbol{A}$ : monodisperse, $\diamond$ : polydisperse $z=3,+: z=1)$. Solid lines refer to Equation (13) (monodisperse) and (14) (polydisperse).

used is long enough as to mimic the behaviour of infinitely long ideal chains.

After generating the random walks, three density profiles were stored: the segment-density profile for polymer chains that were accepted between the two plates (and thus did not cross a plate) $n(x)$, the density of all segments that were generated between the plates $n_{\text {all }}(x)$, and the density profile $n_{\text {single }}(x)$ as if there would only be a single plate. The last two profiles were obtained by using a 'reflecting' boundary condition: once coordinate $x$ of the random walk crosses one of the boundaries, it is forced back to the end point of the simulation region. This corresponds to the assumption that in the bulk of the polymer solution the number of polymer segments crossing a certain 'imaginary' plane is (on average) the same as the number of those entering the simulation region. In the limit of a long enough Monte Carlo run this boundary condition is equivalent to setting to zero the gradient $\partial n(x) / \partial x$ of the bulk-segment concentration at both boundaries (for $n_{\text {all }}(x)$ ) or one of the two boundaries (for $n_{\text {single }}(x)$ ). The profiles $n(x)$ and $n_{\text {single }}(x)$ are then normalized by $n_{\text {all }}(x)$ per lattice point in order to properly normalize the segment densities. In this case the statistical error arising from the fact that different lattice points were visited a different number of times during the Monte Carlo simulation is also made significantly smaller. The profile $n_{\text {single }}(x)$, obtained at large plate separation, is used to evaluate the profile near a single plate. The interaction potential is calculated by integrating the segment density profiles $n(x)$ using Equation (10).
In Figure 1, the Monte Carlo (MC) computer simulation results are plotted (symbols) and are shown to be in very good agreement with exact results for monodisperse polymer and polymer samples with a Schulz chain-length distribution with $z=3$ and $z=1$. The simulations under-predict the interaction potential very slightly. It is noted that there is always a standard deviation in the simulation results. In Figure 2, the Monte Carlo simulation results for the single plate situation are plotted as the symbols, agreeing well with Equation (13) and (14), and thus demonstrating the accuracy of the MC method on the polymer-segment density level.

For the profile between two parallel flat plates we apply the product function Ansat ${ }^{[12]}$ and arrive at:

$f_{\mathrm{p}, \text { tot }}^{\text {poly }}(x)=\frac{\int_{0}^{\infty} \mathrm{d} N \Psi(N) N f_{\mathrm{p}}(x, N) \int_{0}^{\infty} \mathrm{d} N \Psi(N) N f_{\mathrm{p}}(h-x, N)}{\left[\int_{0}^{\infty} \mathrm{d} N \Psi(N) N\right]^{2}}$

In another approximation first the product for each fraction is taken and then the sum over all fractions is taken:

$$
f_{\mathrm{p}, \text { tot }}^{2, \text { poly }}(x)=\frac{\int_{0}^{\infty} \mathrm{d} N \Psi(N) N f_{\mathrm{p}}(h-x, N) f_{\mathrm{p}}(x, N)}{\int_{0}^{\infty} \mathrm{d} N \Psi(N) N}
$$

In Figure 3 full curves are drawn for the profile between the plates according to Equation (15) $(z=3$ and $z=1)$ and for monodisperse polymers (see ref. ${ }^{[12]}$ ) for $h / R_{\mathrm{g}}=7.07$ (a) and $h / R_{\mathrm{g}}=4.24$ (b). The MC results are represented by the symbols. The product function Ansatz of Equation (15) (full curves) agrees well with the simulation results. In Figure $3 \mathrm{~b}$ we have plotted the results with Equation (16) for $h / R_{\mathrm{g}}=4.24$ as the dashed curves. Using Equation (16) instead of (15) hardly affects the final results. It follows that Equation (15) gives a slightly better result for small plate separations and the two approaches are similar for the largest part of plate separations. Equation (15) is therefore used in the rest of this paper. It is noted that the agreement is as satisfying for other chain-length distributions (lognormal; Gaussian). It is clear that less polymer can enter the gap for a wider size distribution for the gap widths, because for a wider distribution there are more large chains with a smaller probability of entering the gap. For very small gap widths it is known that the product function Ansatz overestimates the polymer-segment density (see Figure 2 in ref. $\left.^{[12]}\right)$. This is also the case for polydisperse polymers. The effect of this deviation on the interaction potential is small; the polymer concentration is small in both cases and the total amount of depleted polymer segments is large in both situations $\left(\Gamma\left(h / R_{\mathrm{g}} \rightarrow\right.\right.$ small $) \rightarrow 0$ in any case $)$. An interesting effect was observed for small plate separations, as 

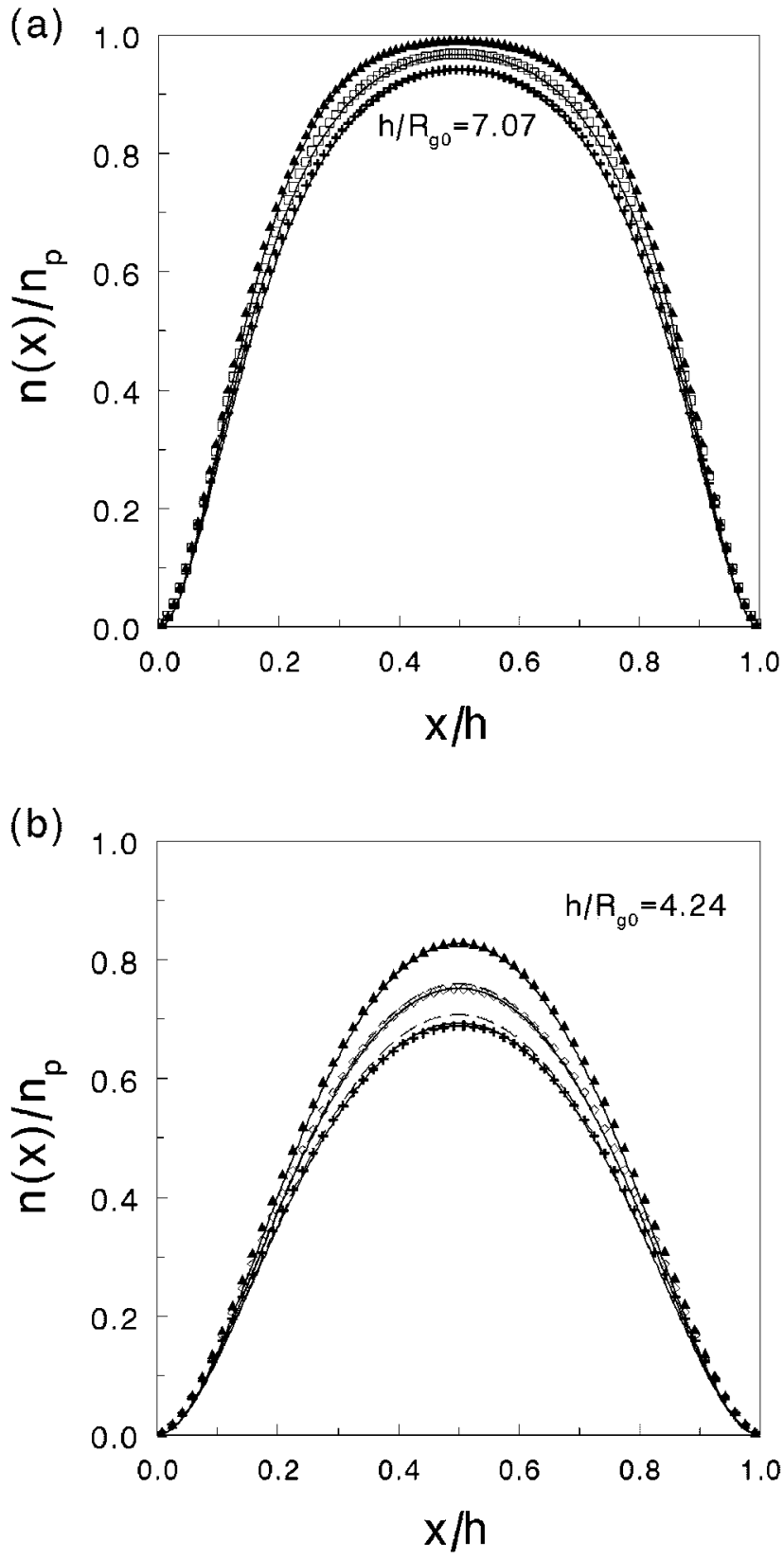

Figure 3. (a) Polymer segment density between two parallel flat plates at $h=7.07 R_{\mathrm{g} 0}$ as a function of the position between the plates for monodisperse ( $\boldsymbol{\Delta})$ and polydisperse polymers $(z=1:+$, $z=3$ : $\square$ ). Full curves follow Equation (15) (polydisperse) or the product function of Equation (13) (monodisperse). (b) As for (a) but at $h=4.24 R_{\mathrm{g} 0}$.

followed from the MC simulation results. For $h / R_{\mathrm{g}}=2.12$ the polymer-segment density is smaller for the more polydisperse polymer sample, see Figure 4 . For $h / R_{\mathrm{g}}=1.41$, however, the opposite was found; more polydisperse polymer enters the gap. This is due to the fact that mainly small polymers $\left(N<N_{0}\right)$ can still enter the gap, which are absent in the monodisperse sample.

Subsequently, we compare the MC chain-length distributions of generated random walks, accepted random walks,

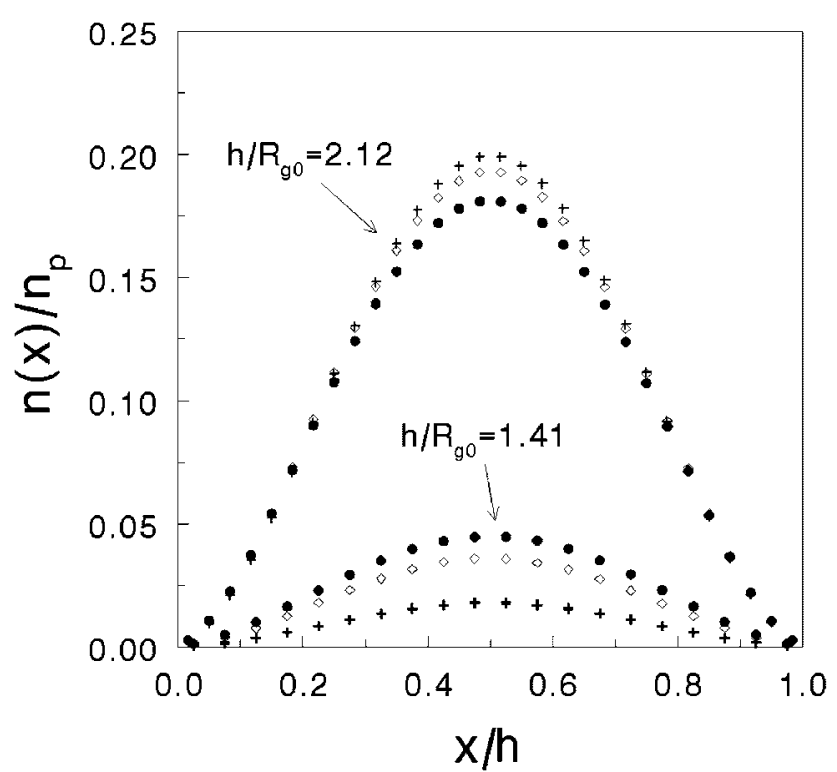

Figure 4. Polymer-segment density between two parallel flat plates at $h=2.12 R_{\mathrm{g} 0}$ and $h=1.41 R_{\mathrm{g} 0}$ as a function of the position between the plates for monodisperse $(+)$ and polydisperse polymers $(z=1: \bigcirc, z=3: \diamond)$ from MC simulations.

and rejected random walks, which are plotted in Figure 5 (Schulz distribution; $z=1, N_{0}=400$ ) for $h / R_{\mathrm{g}}=2.12$ (a) and $h / R_{\mathrm{g}}=1.41$ (b). Naturally, the distributions of generated walks closely follow Equation (8) (full curve) with $z=1$ and $N_{0}=400$. The slight scattering of the points arises from the statistical noise in a particular Monte Carlo realization with a large, but finite generated number of molecules $\left(10^{6}\right)$. It is remarkable that the accepted walks are also very well described by the Schulz distribution with the same value of the parameter $z=1$ but smaller average length, $N_{0}=197$ for $h / R_{\mathrm{g} 0}=2.12$, and $N_{0}=120$ for $h /$ $R_{\mathrm{g} 0}=1.41$ (full curves are best fits following the Schulz distribution). For the rejected walks, the Schulz distribution does not describe the chain-length distributions properly, as is observed in Figure 5a and 5b (best fits are also given as full curves).

The polymer-segment density profiles that can be calculated from the product-function approximation (Equation (15)) yield the interaction potential by inserting it into Equation (11), and Equation (11) into Equation (10). The results for the interaction potential are plotted for monodisperse polymers and for polydisperse polymers for a Schulz distribution with $z=1$ in Figure 6 (symbols). The agreement with the exact results of Equation (5) (full curves) is quite reasonable. Only for intermediate values of $h / R_{\mathrm{g}}$ is there some deviation, which is similar for monodisperse and polydisperse samples. The agreement gives confidence that the product-function approximation is accurate. Hence it is next used to describe the polymersegment density in the space around two spheres in an ideal polydisperse polymer solution. 
(a)
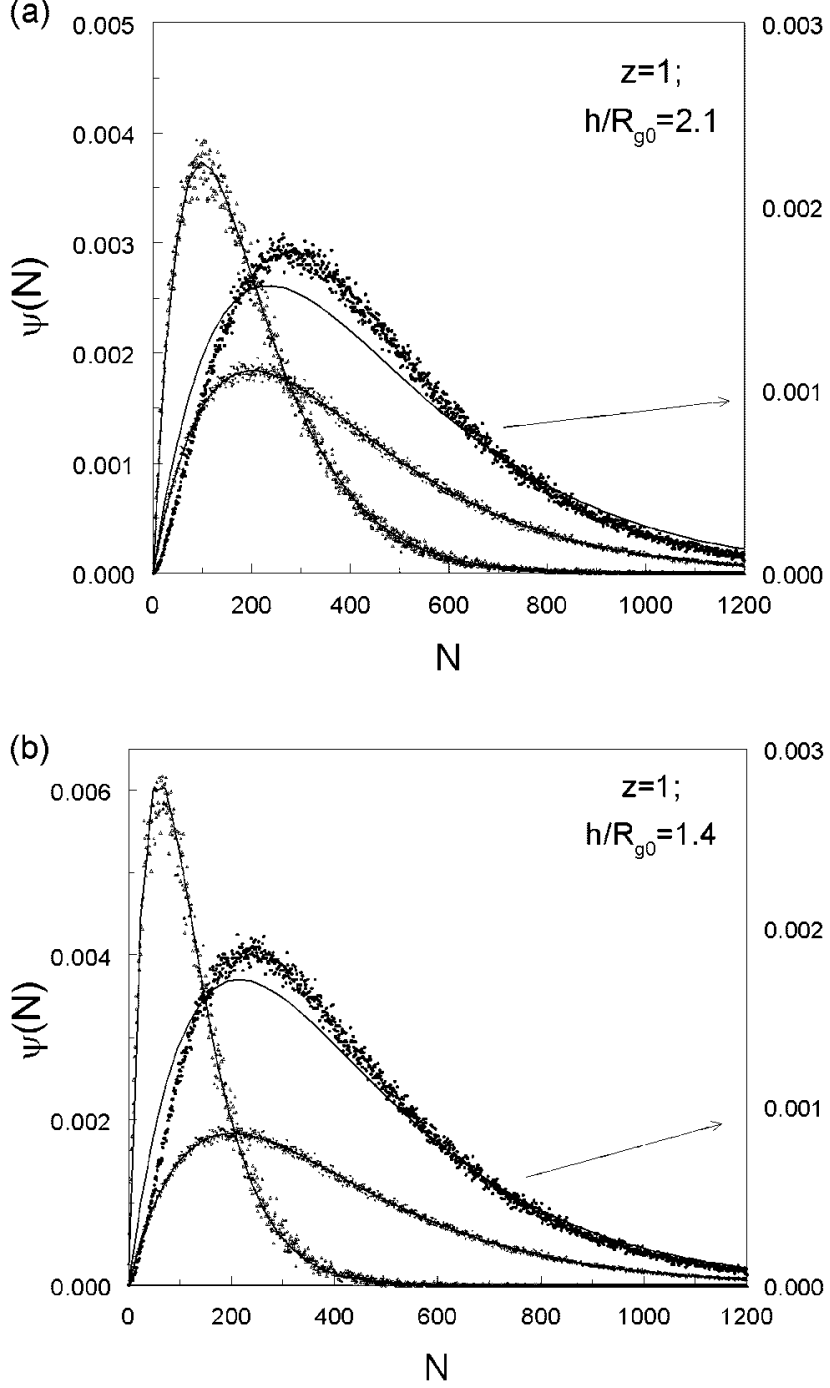

Figure 5. (a) Chain-length distribution from MC simulation of generated random walks $(+)$, accepted random walks $(\triangle)$, and rejected random walks ( $)$; refer to right axis) for $z=1$ and $h=2.12 R_{\mathrm{g} 0}$. The solid lines are best fits of Equation (8). (b) As for (a) but for $h=1.41 R_{\mathrm{g} 0}$.

\section{Polydisperse Ideal Polymer Interacting with Spheres}

\section{Polymer Segment Density Profiles Surrounding Two Spheres}

Within the product-function approach for two spheres immersed in a polydisperse ideal polymer solution we can calculate the local profile using

$f_{\mathrm{s}, \mathrm{tot}}^{\text {poly }}(x)=\frac{\int_{0}^{\infty} \mathrm{d} N \Psi(N) N f_{\mathrm{s}, 1}(\underline{r}, N) \int_{0}^{\infty} \mathrm{d} N \Psi(N) N f_{\mathrm{s}, 2}(\underline{r}-x, N)}{\left[\int_{0}^{\infty} \mathrm{d} N \Psi(N) N\right]^{2}}$

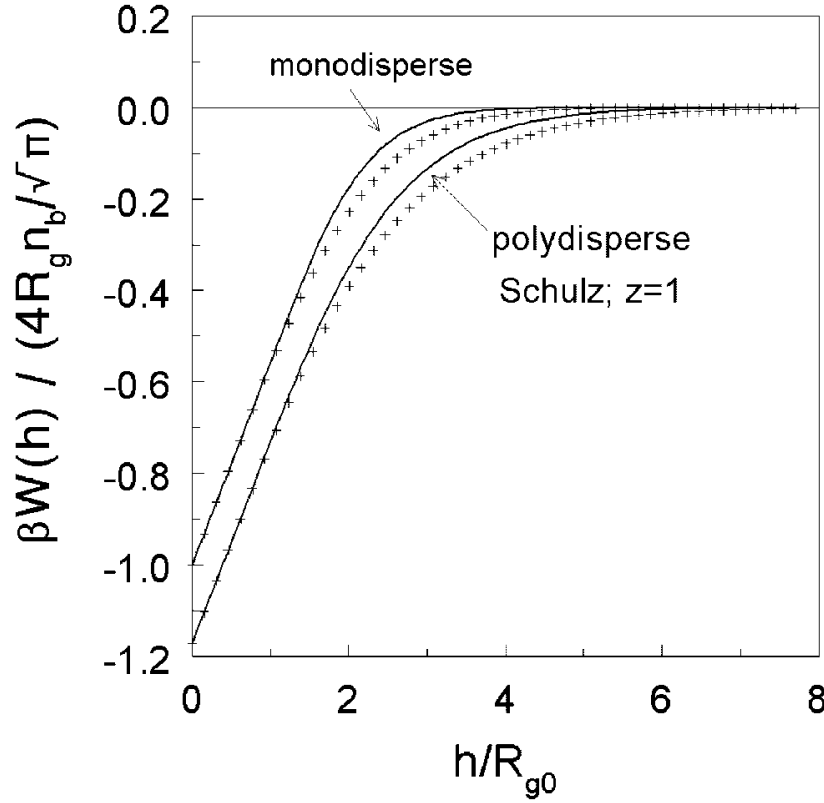

Figure 6. Interaction potential between two plates. Solid lines as in Figure 1 for monodisperse ideal polymers and polydisperse ideal polymers (Schulz, $z=1$ ). Crosses are the predictions from the product-function approximation.

where 1 and 2 refer to the different spheres. The profile, $f_{\mathrm{s}}$, around a single sphere with radius $R$ was calculated by Taniguchi et al. ${ }^{[16]}$ (or see ref. ${ }^{[17]}$ ) and reads

$f_{\mathrm{s}}(x)=\left(\frac{x}{R+x}\right)^{2}\left[1+2\left(\frac{R}{x}\right) A(x)+\left(\frac{R}{x}\right)^{2} B(x)\right]$

with

$$
\begin{aligned}
A(x)= & \operatorname{erf}\left(\frac{x}{2 R_{\mathrm{g}}}\right)-\frac{1}{2}\left(\frac{x}{R_{\mathrm{g}}}\right)^{2}\left(1-\operatorname{erf}\left(\frac{x}{2 R_{\mathrm{g}}}\right)\right) \\
& +\frac{x}{R_{\mathrm{g}} \sqrt{\pi}} \exp \left(-\left(\frac{x}{2 R_{\mathrm{g}}}\right)^{2}\right)
\end{aligned}
$$

and

$$
\begin{aligned}
B(x)= & 2 \operatorname{erf}\left(\frac{x}{2 R_{\mathrm{g}}}\right)-\operatorname{erf}\left(\frac{x}{R_{\mathrm{g}}}\right)+\frac{2}{\sqrt{\pi}}\left(\frac{x}{R_{\mathrm{g}}}\right) \\
& {\left[\exp \left(-\left(\frac{x}{2 R_{\mathrm{g}}}\right)^{2}\right)-\exp \left(-\left(\frac{x}{R_{\mathrm{g}}}\right)^{2}\right)\right] } \\
& +2\left(\frac{x}{R_{\mathrm{g}}}\right)^{2}\left[\frac{1}{2}-\operatorname{erf}\left(\frac{x}{R_{\mathrm{g}}}\right)+\frac{1}{2} \operatorname{erf}\left(\frac{x}{2 R_{\mathrm{g}}}\right)\right]
\end{aligned}
$$

where the range of the profile now depends on the ratio $R / R_{\mathrm{g}}$ and goes to $f_{\mathrm{p}}(x)$ (Equation (13)) for $R / R_{\mathrm{g}} \rightarrow \infty$ (note that $B(x)$ is the flat wall result). By applying Equation (12) the profiles around two spheres can be computed, which for monodisperse polymers have been shown to be quite accurate. $^{[12]}$ 
The simulation of random walks in the space surrounding two spheres was performed in cylindrical symmetry, with the symmetry axis connecting the centers of the spheres. The problem is then mapped onto a two-dimensional lattice with coordinates $y$ along the symmetry axis and $x$ representing the distance from the axis of rotation. One polymer molecule is represented by performing a random walk with $N_{2 D}$ steps, which is a fixed number in the monodisperse case, or is generated randomly using the Schultz distribution (Equation (8)) for the polydisperse case (typically, $\left\langle\mathrm{N}_{2 \mathrm{D}}\right\rangle=N_{0}=400$ ). Since in this case the walks are performed in two dimensions, the radius of gyration in two dimensions, $R_{\mathrm{g}}^{2 \mathrm{D}}$ equals $\sqrt{ }\left(\left\langle N_{2 \mathrm{D}}\right\rangle / 4\right)$. These steps are movements along either $y$, with a probability of $25 \%$ in both the negative and positive direction, or along $x$, with a probability of $x /(4 x+2)$ to reduce $x$ (negative $x$ values are impossible) by 1 or a probability of $(x+1) /(4 x+2)$ to increase $x$. The difference of probabilities between the upward and downward movements along $x$ accounts for a proper use of the phase space in the curved cylindrical coordinates. The 'reflecting' boundary conditions, as described above for the one-dimensional Monte Carlo scheme for the case of two plane walls, are applied at the limiting values of $y$ and $x$ at the boundary of the simulation region.

The following procedure was used to choose the starting point $\left(x_{0}, y_{0}\right)$ of the random walk. In general, in the curved cylindrical coordinates the probability of finding the first segment of the trial molecule at the coordinate $x_{0}$ should linearly increase with $x_{0}$ because of the increase of the volume associated with one lattice point. If one were to plainly incorporate this into the simulation program, most of the computer time will be spent simulating molecules at large values of $x$, where the depletion effects are absent. Only a very small fraction of the computers efforts will then be spent simulating the segment density at small $x$, the area of most interest for the depletion between two spheres with radius $R$ small compared to the total size $x_{\max }$ of the simulation area in the $x$ direction. The condition $x_{\max } \gg R$ is needed to minimize the size artifacts of the simulation scheme. To achieve a more efficient Monte Carlo scheme, the starting point $\left(x_{0}, y_{0}\right)$ of the random walk is randomly chosen with equal probabilities for all points on the $(x, y)$ plane. The introduced non-uniformity of the distribution of the starting points is compensated by giving a weight equal to $\left(2 x_{0}+1\right)$ to the whole walk, which recovers the uniform bulk concentration of the segments in the limit of a long enough Monte Carlo run. The program then spends much more time in the region of interest, and hence reduces the statistical uncertainty considerably. A typical simulation result of the polymer-segment density around two spheres is given in Figure 7, where the gray scale represents the polymer-segment density (black: no polymer; white: bulk polymer-segment density).

The program is able to simulate the depletion interactions between two spheres, but also, for instance, between a

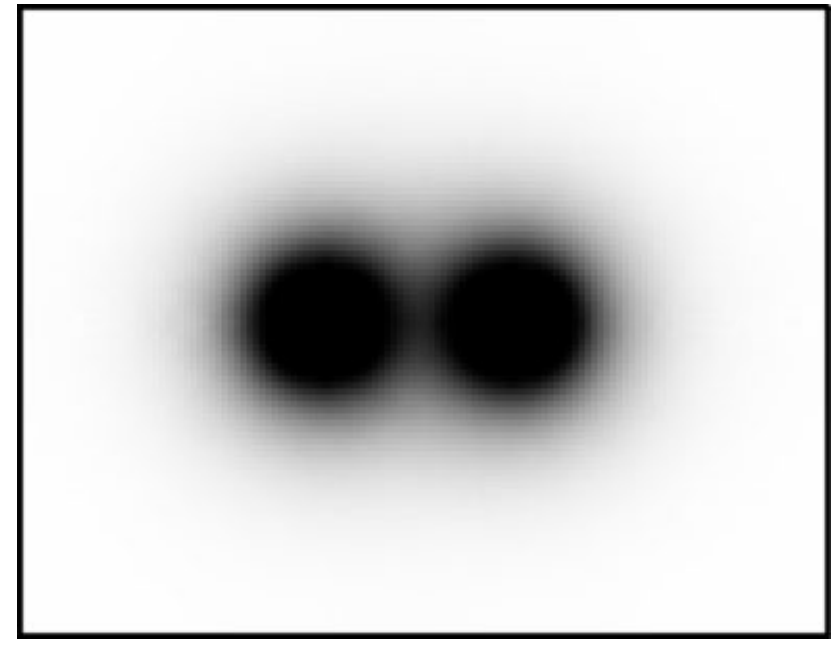

Figure 7. MC simulation snapshot of the density of polymer segments around two identical spheres with radius $R=R_{\mathrm{g} 0}$. The polymer is polydisperse and the chain-length distribution is given by the Schulz distribution with $z=1$. The gray scale is proportional to the polymer-segment density (black: $n=0$, white: $\left.n=n_{\mathrm{p}}\right)$.

sphere and a plane. In the following we focus on comparing the $\mathrm{MC}$ and product-function results for two spheres (of the same size) only. Similar agreement is found for other geometries as well. In addition, we tested the product-function results against Monte Carlo simulations for polydisperse polymers with distribution of chain length given by lognormal distribution with various widths (not shown here).

In Figure $8 \mathrm{a}$ and $8 \mathrm{~b}$ we present some indicative polymersegment density profiles for monodisperse and polydisperse $(z=1)$ polymer from MC simulation results as compared to Equation (17) for polydisperse polymer $(z=1$; dashed curves) and Equation (18) for monodisperse polymer (full curves). The position halfway between the two spheres defines where both $x$ and $y$ are 0 . In Figure 8a $n(y) / n_{\mathrm{p}}$ is plotted as a function of $y$ for three values of $x ; x /$ $R_{\mathrm{g} 0}=1.2,1.8$, and 2.4 , for $h / R_{\mathrm{g} 0}=0.48$ and $R=R_{\mathrm{g} 0}$. The MC simulation data are given as symbols (monodisperse: filled, $z=1$; open). The MC simulation densities agree well with the product function Ansatz, although the product function slightly underestimates the polymer densities. For $h / R_{\mathrm{g} 0}=0$ and $R=R_{\mathrm{g} 0}$ and similar values of $x / R_{\mathrm{g} 0}$, the results are plotted in Figure $8 \mathrm{~b}$. Here the agreement is also satisfying. In addition, we found that for $R_{\mathrm{g} 0} / R=3$ and $1 / 3$ that the agreement is very satisfying.

\section{Interaction Potential between Two Spheres}

The agreement between the product-function approximation and the MC simulation results motivates the comparison of results also on the level of the pair interaction. The interaction potential can be directly computed from the 

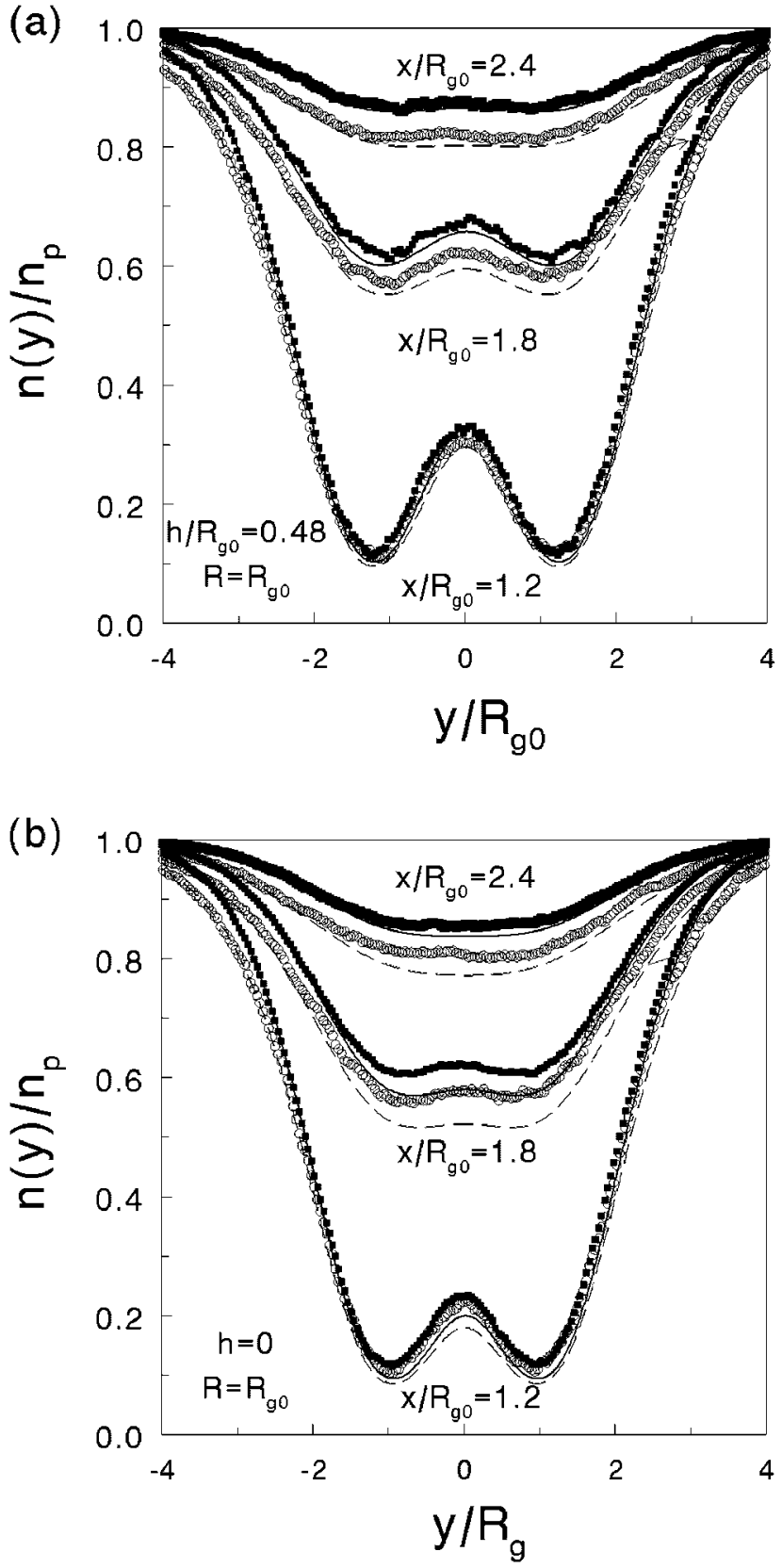

Figure 8. (a) Polymer-segment density as a function of $y$ for three values of $x$, as indicated, in the space surrounding two colloidal spheres with radius $R=R_{\mathrm{g} 0}$ and $h=0.48 R_{\mathrm{g} 0}$. Symbols are the MC results: polydisperse polymer $(\bigcirc ; z=1)$ and monodisperse polymer $(\mathbf{O})$ samples. Curves are the predictions of the product-function approximation for monodisperse polymer (solid lines) and polydisperse polymer $(z=1$, dashed lines). (b) As for (a) but for $h=0$.

density profiles by inserting them into Equation (11) and (10). The results for $R=R_{\mathrm{g} 0}$ are plotted in Figure 9. The symbols are the MC simulation data (standard deviation was always smaller than $0.5 \mathrm{k} T R_{\mathrm{g} 0}^{3} n_{\mathrm{b}}$ ), through which the dashed curves are drawn to guide the eye. The productfunction approximation yields the full curves. The results

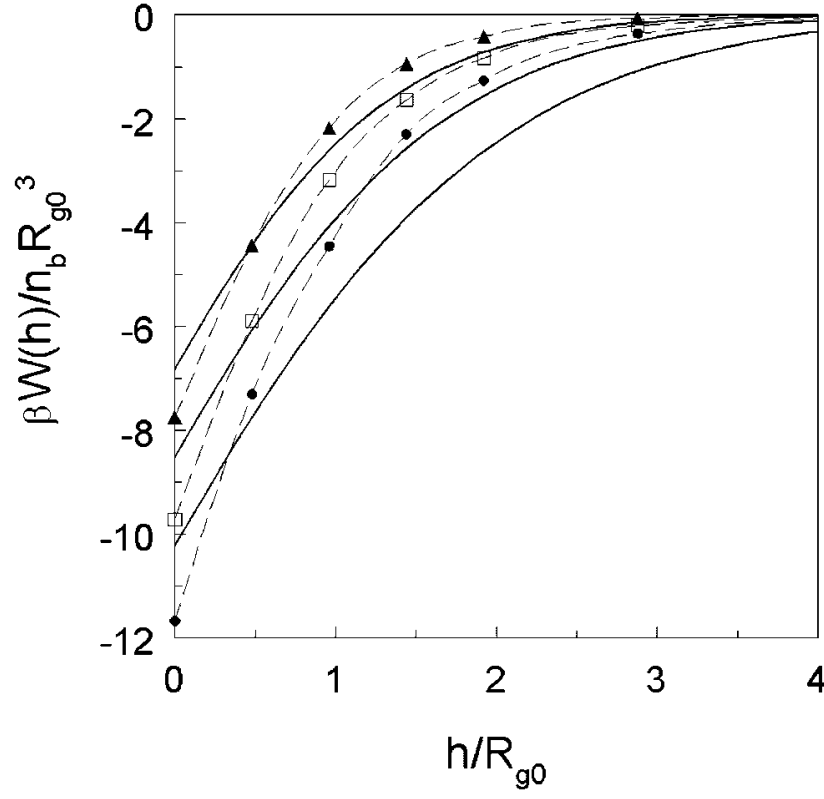

Figure 9. Interaction potential between two spheres with radius $R=R_{\mathrm{g} 0}$ as a function of the distance between them in ideal monoand polydisperse polymer solutions. Full curves are the predictions of the product function approximation (from top to bottom: monodisperse, polydisperse $z=3$, and polydisperse $z=1$ ). Symbols refer to the MC results. Dashed curves are plotted to guide the eye.

are comparable with the MC simulation results with respect to the order of magnitude but differs from it in two ways. Firstly, the range of the potential is significantly overestimated by the product function Ansatz. This was already the case for the potential between two plates, but is more pronounced here because the deviations are now integrated in three dimensions. This leads to an overestimation of the potential for most interparticle distances $h$. Obviously a slight overestimation of the polymer densities as in Figure 8 lead to more significant deviations when all densities are integrated in three dimensions. Secondly, the depth of the sphere-contact potential is underestimated somewhat by the product-function approximation. This effect may be because of the small overestimation of the polymersegment density profiles when the colloidal surfaces are very near one another. Still, in order to estimate the effects of polydisperse polymer the product-function approximation provides an easy reasonable estimate of the potential curve and a good estimate for the detailed density profiles around the spheres. In general, a more polydisperse sample leads to a slightly longer-ranged attraction and to a somewhat deeper potential. From the simulation results it follows that the contact potential is about 1.5 times as much for $z=1$ as for the monodisperse case. The effect of polydispersity on the range and strength of the interaction between two spheres is therefore larger as compared to its effect on the potential between two plates. The longerranged potential may affect the observed phase behavior in 
the sense that polydispersity of the polymer enhances gasliquid coexistence to dominate with respect to gas-solid coexistence in experimental studies ${ }^{[2]}$ in comparison with theoretical predictions. ${ }^{[18-20]}$

A simple way to obtain a reasonable estimation of the effects of polydispersity on the depletion interaction between two spheres in a solution of ideal polymer chains is by insertion of the polydisperse depletion thickness, $\Delta_{\mathrm{pol}}$, in the classical Asakura-Oosawa ${ }^{[21]}$ expression

$W(h)=-\frac{2}{3} \pi n_{\mathrm{b}} \Delta_{\mathrm{pol}}^{3}\left(1-\frac{h}{2 \Delta_{\mathrm{pol}}}\right)^{2}\left(2+\frac{3 R}{\Delta_{\mathrm{pol}}}+\frac{h}{2 \Delta_{\mathrm{pol}}}\right)$

for $h \leq 2 \Delta_{\text {pol }}$, and $W(h)=0$ for $h>2 \Delta_{\text {pol }}$, see also Vrij. ${ }^{[22]}$ In the derivation of Equation (19) it was assumed that the depletion layers could be replaced by simple step-functions. The depletion thickness of polydisperse ideal polymers could be obtained by inserting the depletion layer thickness for monodisperse polymers around a sphere, given by

$$
\frac{\Delta}{R}=\left(1+\frac{6 q}{\sqrt{\pi}}+3 q^{2}\right)^{1 / 3}-1
$$

(see Equation (A4) in ref. ${ }^{[20]}$ ), into Equation (21)

$$
\Delta_{\mathrm{pol}}=\frac{\int_{0}^{\infty} \mathrm{d} N \Delta \Psi(N) N}{\int_{0}^{\infty} \mathrm{d} N \Psi(N) N}
$$

which turns to Equation (7) (the flat wall case) in the limit $q \rightarrow 0$. In Figure 10 we plot the minimum of the interaction potential for a wide range of polymer/colloid size ratios for monodisperse and polydisperse (Schulz distribution with $z=1$ and 3) as follows from the MC simulation results (symbols) and the simple theory following Equation (19) (full curves). The contact potential dependence on polydispersity is weak for relatively long polymer chains. It seems that for the interaction between relatively small spheres size polydispersity of the large polymer chains hardly matters, and that the contact potential tends towards a universal $q$-dependent scaling for very small spheres. Equation (19) describes the contact potential reasonably well. It is noted that it underestimates the range of the potential to some extent.

\section{Conclusions}

A theory was proposed for the interaction between colloidal particles due to non-adsorbing ideal polydisperse polymers by extending the product function Ansatz. Monte Carlo computer simulations were performed to test the theory. For two parallel flat plates immersed in an ideal polydisperse polymer solution it followed that the product function Ansatz gives a good description of the polymer-segment

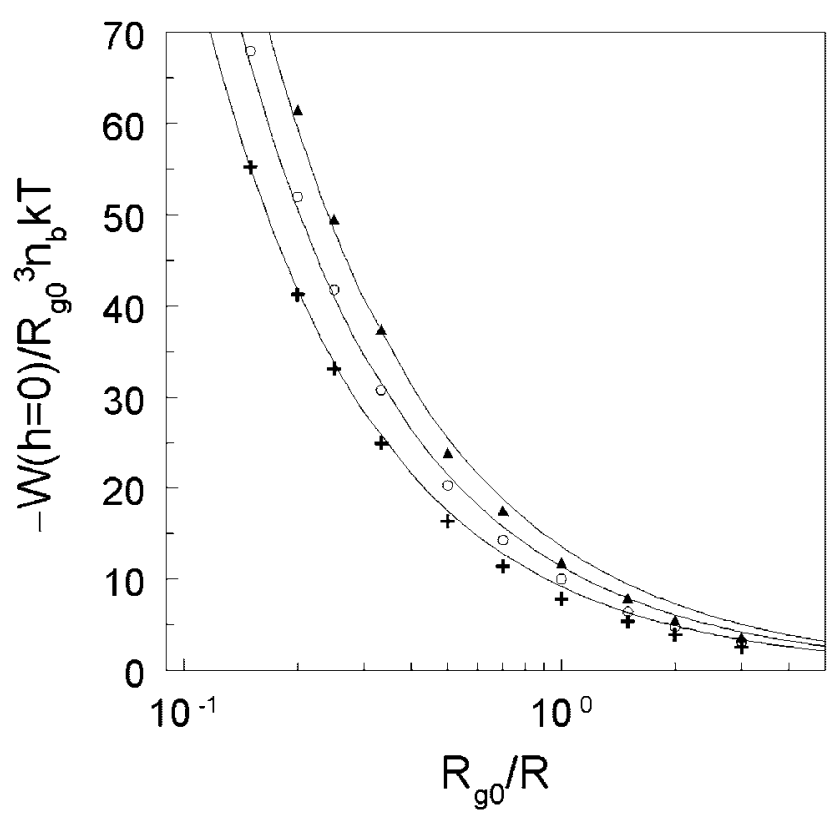

Figure 10. Contact potential between two spheres in a nonadsorbing ideal polymer solution as a function of the size ratio $R_{\mathrm{g}} /$ $R$ for monodisperse polymer ( + : MC simulation results) and polydisperse polymer with a Schulz distribution; $z=1(\boldsymbol{\Delta})$ and $z=3(\bigcirc)$. Symbols refer to the MC simulation results and full curves to the predictions using Equation (19).

density between the plates and of the interaction potential. In addition, the predicted polymer densities of ideal polydisperse polymers around two spheres agree well with the simulation results. For the interaction between two spheres, the product function Ansatz predicts reasonable values for the minimum of the interaction potential induced by the polydisperse polymers, but the range of the potential is overestimated somewhat by the proposed analytical theory. Polydispersity increases the range of the interaction potential, the depth of the potential minimum, and enhances size fractionation. The effects of polydispersity on the interaction potential between two plates are weak but are stronger between two (large) spheres. A simple analytical prediction of the effects of polydispersity for the pair interaction of two spheres is given for a first estimation of the effects.

\section{Appendix}

The depletion layer thickness near a single plate due to polydisperse polymer can be calculated in two ways. Firstly, using Equation (7). Secondly, we can first calculate the polymer-segment density profile of polydisperse polymer chains near a single particle given for a plate as

$$
f_{\mathrm{p}}^{\mathrm{pol}}(x)=\frac{\int_{0}^{\infty} \mathrm{d} N \Psi(N) N f_{\mathrm{p}}(x, N)}{\int_{0}^{\infty} \mathrm{d} N \Psi(N) N}
$$


in analogy with Equation (15) and then integrate the profile using

$$
\Delta_{\mathrm{pol}}=\int_{0}^{\infty} \mathrm{d} x\left[1-f_{\mathrm{p}}^{\text {poly }}(x)\right]
$$

which gives exactly the same result as Equation (5).

Acknowledgement: J. K. G. Dhont and H. N. W. Lekkerkerker are thanked for giving us the opportunity to finish this work. We thank D. G. A. L. Aarts for a critical reading of the manuscript.

Received: July 15, 2002

Revised: October 15, 2002

Accepted: October 16, 2002

[1] W. C. K. Poon, Curr. Opin. Colloid Interface Sci. 1998, 3, 593.

[2] S. M. Ilett, A. Orrock, W. C. K. Poon, P. N. Pusey, Phys. Rev. E: Stat. Phys., Plasmas, Fluids, Relat. Interdiscip. Top. 1995, $51,1344$.

[3] N. A. M. Verhaegh, J. S. Van Duijneveldt, J. K. G. Dhont, H. N. W. Lekkerkerker, Phys. A (Amsterdam, Neth.) 1996, $230,409$.
[4] I. Bodnar, J. K. G. Dhont, H. N. W. Lekkerkerker, J. Phys. Chem. 1996, 100, 19614.

[5] E. H. A. De Hoog, H. N. W. Lekkerkerker, J. Phys. Chem. B 1999, 103, 5274.

[6] P. B. Warren, Langmuir 1997, 13, 4388.

[7] R. P. Sear, D. Frenkel, Phys. Rev. E: Stat. Phys., Plasmas, Fluids, Relat. Interdiscip. Top. 1997, 55, 1677.

[8] M. Peich, J. Y. Walz, J. Colloid Interface Sci. 2000, 225 , 134.

[9] D. Goulding, J.-P. Hansen, Mol. Phys. 2001, 99, 865.

[10] Y. Mao, M. E. Cates, H. N. W. Lekkerkerker, Phys. A (Amsterdam, Neth.) 1995, 222, 10.

[11] S. G. Ash, D. H. Everett, C. Radke, J. Chem. Soc., Faraday Trans. 1973, 69, 1256.

[12] R. Tuinier, G. A. Vliegenthart, H. N. W. Lekkerkerker, J. Chem. Phys. 2000, 113, 10768.

[13] R. Tuinier, G. A. Vliegenthart, H. N. W. Lekkerkerker, Macromolecules 2001, 34, 4636.

[14] S. Asakura, F. Oosawa, J. Chem. Phys. 1954, 22, 1255.

[15] E. Eisenriegler, J. Chem. Phys. 1983, 79, 1052.

[16] T. Taniguchi, T. Kawakatsu, K. Kawasaki, AIP Conf. Proc. 1992, 256, 503.

[17] E. Eisenriegler, A. Hanke, S. Dietrich, Phys. Rev. E: Stat. Phys., Plasmas, Fluids, Relat. Interdiscip. Top. 1996, 54, 1134.

[18] A. P. Gast, C. K. Hall, W. B. Russel, J. Colloid Interface Sci. 1983, 96, 251.

[19] H. N. W. Lekkerkerker, W. C. K. Poon, P. N. Pusey, A. Stroobants, P. B. Warren, Europhys. Lett. 1992, 20, 559.

[20] D. G. A. L. Aarts, R. Tuinier, H. N. W. Lekkerkerker, J. Phys.: Condens. Matter 2002, 14, 7551.

[21] S. Asakura, F. Oosawa, J. Polym. Sci. 1958, 33, 183.

[22] A. Vrij, Pure Appl. Chem. 1976, 48, 471. 